\title{
Stratégies paysannes pour la protection du maïs indigène dans l'État de Tlaxcala
}

Peasant strategies for the protection of native corn in the State of Tlaxcala Estrategias campesinas para la protección de los maíces nativos en el estado de Tlaxcala

\section{Pánfilo Hernández Ortiz}

\section{CpenEdition}

\section{Journals}

Édition électronique

URL : https://journals.openedition.org/ethnoecologie/7565

DOI : $10.4000 /$ ethnoecologie. 7565

ISSN : 2267-2419

Éditeur

Laboratoire Éco-anthropologie

Référence électronique

Pánfilo Hernández Ortiz, « Stratégies paysannes pour la protection du maïs indigène dans l'État de Tlaxcala », Revue d'ethnoécologie [En ligne], Supplément 2 | 2021, mis en ligne le 25 novembre 2021, consulté le 20 décembre 2021. URL : http://journals.openedition.org/ethnoecologie/7565 ; DOI : https://doi.org/10.4000/ethnoecologie.7565

Ce document a été généré automatiquement le 20 décembre 2021. 


\section{Stratégies paysannes pour la protection du maïs indigène dans l'État de Tlaxcala}

Peasant strategies for the protection of native corn in the State of Tlaxcala Estrategias campesinas para la protección de los maíces nativos en el estado de Tlaxcala

Pánfilo Hernández Ortiz

\section{Introduction}

1 Les transformations éprouvées par le Mexique depuis son entrée dans l'Accord de Libre-Échange Nord-Américain (ALENA) en 1994 ont été diverses. L'ouverture des frontières aux produits agricoles a eu de profondes conséquences dans le monde rural. Le maïs nord-américain à prix réduit, obtenu grâce à une agriculture intensive et extensive largement subventionnée par le gouvernement, a généré d'importants déséquilibres dans les campagnes mexicaines. Ne pouvant pas concurrencer ces prix, les paysans mexicains ont quitté, dans de nombreux cas, leurs parcelles ou abandonné leurs pratiques agricoles traditionnelles pour adopter des semences améliorées, de la machinerie agricole et des produits agrochimiques.

2 Cependant, les effets de telles transformations ont été souvent négatifs. Les rendements n'ont pas été ceux espérés et les profits n'ont pas été suffisants pour payer les dépenses que ce nouveau modèle agricole nécessitait. De plus, l'usage des semences améliorées a provoqué un remplacement progressif des variétés locales de maïs et, de ce fait, une érosion de la diversité génétique.

Dans ce contexte, le Grupo Vicente Guerrero (GVG) a déployé diverses initiatives dans l'État de Tlaxcala cherchant à empêcher la substitution des variétés locales de maïs par des semences hybrides et, plus tard, par les transgéniques. À ce sujet l'expérience du GVG est intéressante non seulement par le fait que le Groupe a réussi à faire passer, en 
2011, la « Loi de promotion et de protection du maïs comme patrimoine originaire, en constante diversification et alimentaire pour l'État de Tlaxcala ", mais aussi parce que son travail auprès des communautés a encouragé des processus d'organisation communautaire. Ainsi, le GVG a provoqué une prise de conscience des risques posés par l'utilisation de semences transgéniques. Face à cela, des dynamiques pour revaloriser et échanger des semences paysannes entre les différentes communautés se sont développées. Il convient de noter que vue leur efficacité, ces initiatives sont aujourd 'hui reproduites dans d'autres régions du pays.

Ainsi, cet article propose quatre sections et quelques conclusions. Dans la première nous expliquons l'importance du maïs pour l'économie et la société au Tlaxcala. Dans la seconde section nous abordons l'histoire du Grupo Vicente Guerrero depuis les premières expériences de ses promoteurs à la fin des années 1970, en passant par leur participation dans l'association civile « Programme de développement rural de service, développement et paix » (Programa de Desarrollo Rural de Servicio, Desarrollo y Paz, A.C., SEDEPAC), jusqu'à leur participation aux réunions «Paysan à Paysan " (Campesino $a$ Campesino) tenues au Mexique et dans d'autres pays d'Amérique Latine. Dans cette seconde section, nous racontons aussi le travail du GVG sur l'amélioration des variétés de maïs dans les communautés rurales de Tlaxcala. La troisième section rend compte des actions déployées par le GVG à partir de 2000 pour contenir l'entrée de semences hybrides et transgéniques dans la région, ainsi que pour promouvoir la protection des variétés locales et des connaissances et pratiques associées à sa consommation et à son utilisation agricole. Dans ce cas, il est intéressant de noter que c'est par diverses stratégies, notamment juridiques, et des mobilisations avec d'autres groupes, qu'il a été possible, à la fois, de rendre visibles les risques entraînés par l'érosion de la biodiversité cultivée et de mettre en œuvre des stratégies pour agir contre ce problème. Pour ce faire ont été organisées les «foires de semences » (ferias de semillas) comme des espaces pour échanger des semences de ferme et des expériences. La quatrième section montre les résultats obtenus par le GVG par son travail à Tlaxcala et fait le point sur les actions à mener dans le futur proche. Enfin, dans la dernière section sont proposées quelques conclusions.

\section{Le maïs et les paysans de Tlaxcala}

5 Le Mexique est considéré comme le centre d'origine, de diversification et de domestication du maïs. Son nom scientifique est Zea mays et son ancêtre sauvage est le Teocintle. Les premiers vestiges archéologiques de cette plante ont été retrouvés dans la vallée de San Juan de Tehuacán dans l'État de Puebla et datent de 7000 BC. Cependant, d 'autres vestiges de maïs ont été localisés dans les vallées centrales de Oaxaca où se trouve le plus grand nombre de races de maïs (environ 17 types), puis dans le bassin du Balsas qui traverse les États de Guerrero et Michoacán (Kato Yamakake et al. 2013).

Bien que la domestication du maïs ait eu lieu depuis des milliers d'années, il est important de souligner que sa diversification est un processus qui continue aujourd'hui grâce au travail des communautés indigènes et paysannes. Actuellement, 59 races et près de 1200 variétés de maïs ont été reconnues au Mexique, et chacune est adaptée à des conditions agronomiques spécifiques, ainsi qu'aux goûts et besoins des communautés qui les cultivent (Ortega Paczka 2003). À présent, bien que la culture du maïs soit pratiquée dans tout le Mexique, c'est dans les États de Chiapas, Oaxaca, 
Guerrero, Michoacán, Puebla, Jalisco et Tlaxcala que les communautés paysannes et indigènes conservent le mieux les diverses races de maïs. (Figure 1)

Figure 1 : Le Mexique centre d'origine et de diversification du maïs

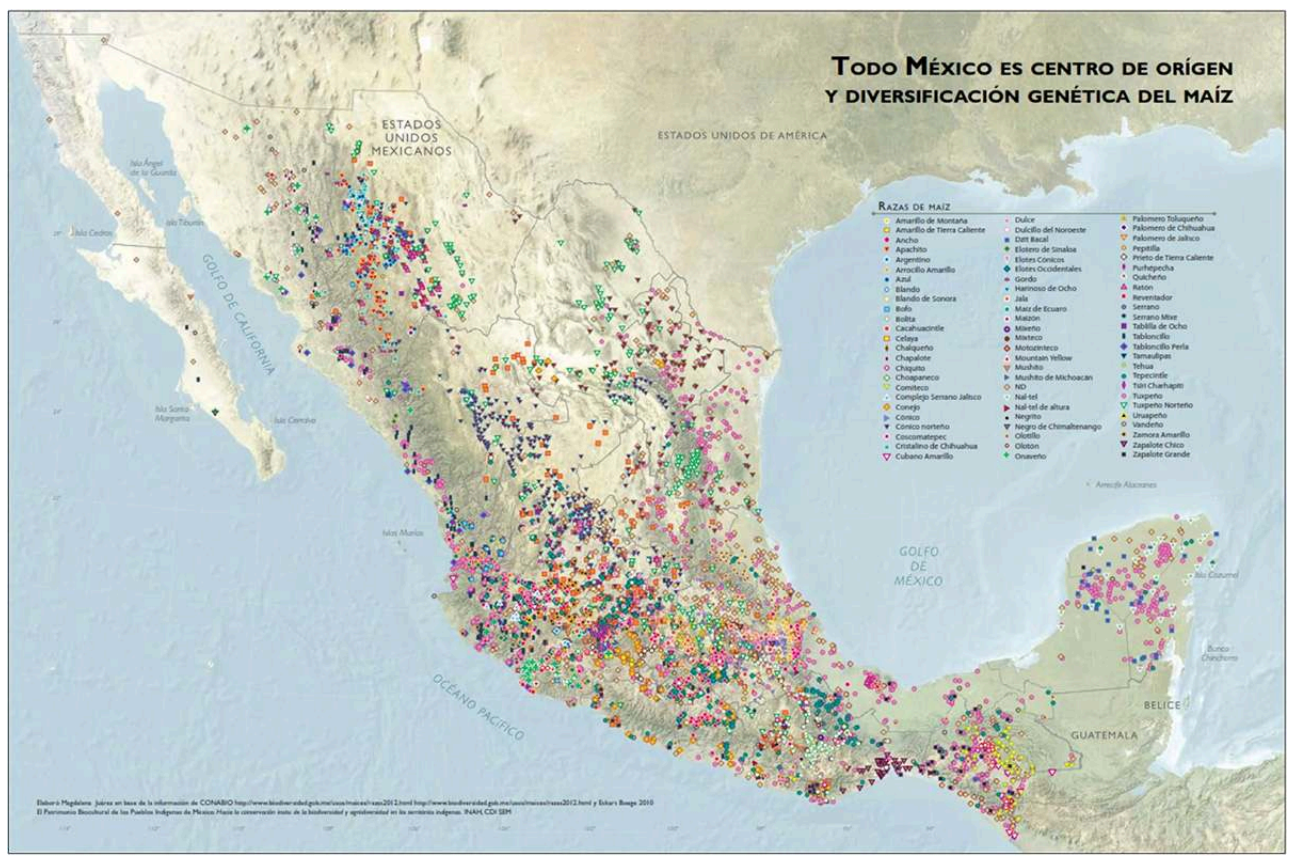

7 Le maïs est une culture fondamentale au Tlaxcala depuis l'époque préhispanique. Selon certains auteurs, le nom même de l'État serait associé au maïs car en langue nahuatl, Tlaxcallan (Tlaxcal ou tlaxcali) signifie lieu de pain de maïs ou des tortillas (Massieu Trigo 2016). Selon les données issues des travaux archéologiques et historiques, le maïs aurait été le principal soutien de l'économie des quatre seigneuries (Tepeticpac, Ocotelulco, Quiahuiztlan et Tizatlán) qui se sont développées dans le territoire de l'actuel État de Tlaxcala. En utilisant un bâton de plantation dénommé coa, les Tlaxcaltecas cultivaient du maïs, des haricots, des courges, des tomates, des agaves et de la cochinilla (Dactylopius coccus), un insecte très apprécié pour fournir une belle teinture rouge. Ces différentes cultures étaient la base de leur économie et, ils la complémentaient avec la cueillette de plantes, herbes et fruits sauvages, à feuilles alimentaires.

Or le maïs n'était pas seulement fondamental pour l'alimentation, mais aussi pour la vie sociale et culturelle. Dans ce sens, il faut observer que le maïs a alimenté le monde symbolique tlaxcaltèque et qu'il a aussi contribué à façonner les pratiques sociales dans la région. Ainsi, compte tenu de son rôle central pour la subsistance et pour la reproduction culturelle de la communauté, le maïs s'est répandu en même temps que les formes de consommation se sont diversifiées. À présent, il est possible de trouver 16 races de maïs qui sont en permanente évolution par leur contact avec les variétés des parcelles voisines. Bien que les races prédominantes dans la région soient les cónicos, chalqueño et cacahuacintle, il est aussi possible de trouver les arrocillo jaune, palomero toluqueño, Celaya, pepitilla, tunnicata et dulce, ainsi qu'une importante diversité de variétés telles que les olote rojo, crema, cristalino, pinto, cañuelilla, xocoyul, negro, azul, rojo, et moradillo parmi d'autres (Ortiz Báez et al. 2016). (Figure 2) 
Figure 2 : Le maïs à Tlaxcala

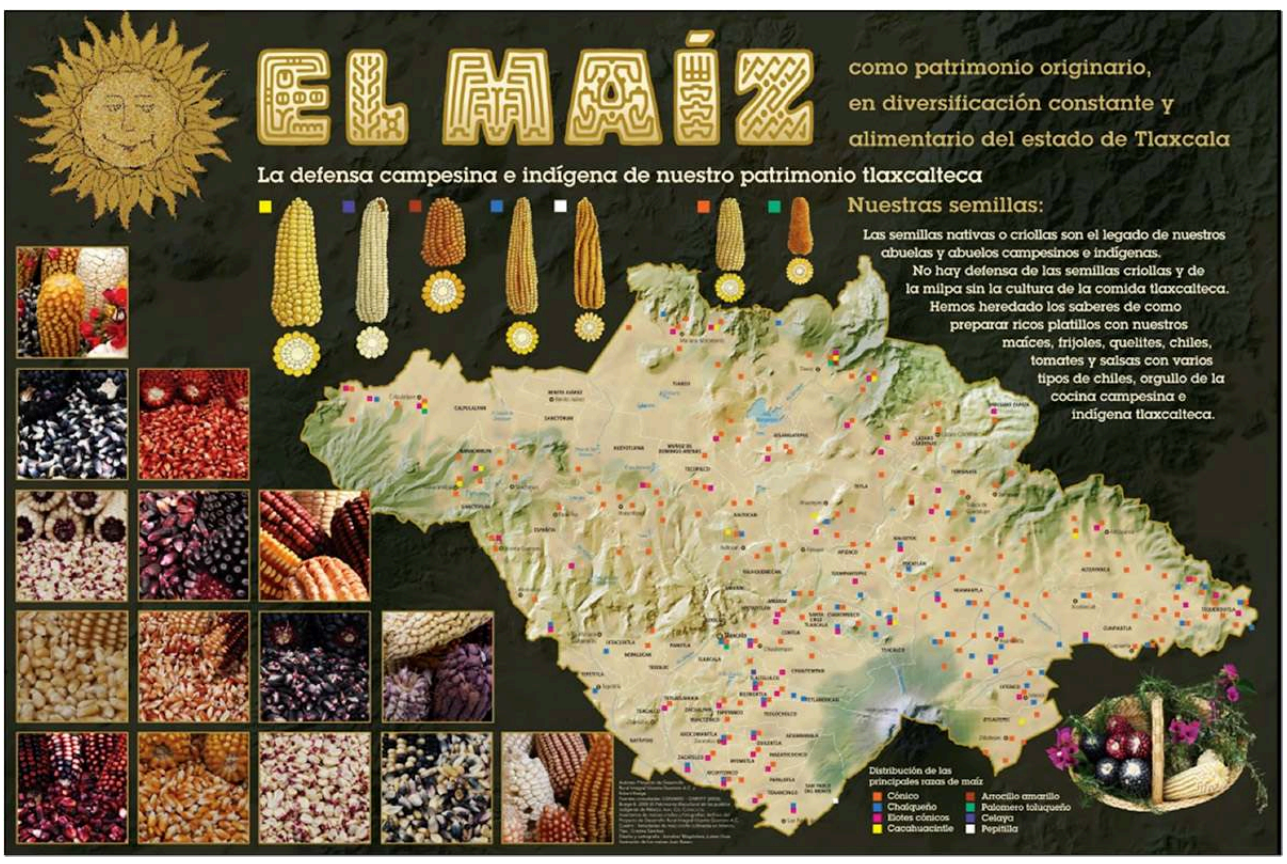

\section{Le Grupo Vicente Guerrero}

9 Le Grupo Vicente Guerrero (GVG) est une organisation paysanne qui promeut le développement durable pour faire face à la pauvreté rurale et à la dégradation de l'environnement. Dans le contexte national, l'action du GVG a été très importante pour la préservation du maïs criollo et pour le développement d'initiatives promouvant une agriculture durable par des stratégies agro-écologiques et l'organisation horizontale et autonome des paysans. Actuellement, le GVG participe à plusieurs initiatives nationales qui rejettent l'entrée du maïs transgénique dans le pays, ainsi qu'à diverses campagnes pour la défense des semences de ferme et des savoir-faire paysans.

Le travail actuellement déployé par le GVG dérive de l'expérience accumulée depuis plus de 40 ans. Les origines du GVG remontent à la fin des années 1970 lorsque l'environnementaliste Rogelio Cova Juárez travaillait à développer des systèmes de production agricole durables dans les communautés de la région. À ce moment-là, Rogelio Cova cherchait à rapprocher les paysans tlaxcaltèques des expériences de conservation des sols et des eaux développées au Guatemala. Dans ce cadre, quatre paysans de la communauté Vicente Guerrero sont partis à Chimaltenango, au Guatemala, où des promoteurs locaux leur ont transmis leurs savoir-faire. Par la suite, à leur retour à Tlaxcala, ces paysans sont devenus des promoteurs actifs de l'agriculture durable et ont aidé à développer la culture fruitière et l'horticulture bio intensive dans l'ejido, ainsi qu'à la création d'une parcelle scolaire dans la communauté.

11 Un peu plus tard, en 1983, ces promoteurs ont rejoint le Programa de Desarrollo Rural de Servicio, Desarrollo y Paz, A. C. (SEDEPAC) où ils ont pu apporter leur expérience dans $\mathrm{d}$ 'autres communautés de Tlaxcala et de Michoacán. Ils ont également promu des camps d'été avec l'aide du SEDEPAC où de jeunes étudiants nord-américains se portaient volontaires pour travailler dans plusieurs communautés du Mexique. Grâce à ces initiatives, les promoteurs ont réussi à revaloriser la milpa traditionnelle, des plantes 
comme le maguey et d'autres plantes médicinales. Ils ont également entrepris des campagnes de reboisement, l'élevage d'espèces de basse-cour et la préparation du compost.

Dans les années 1980, concerné par les problèmes d'approvisionnement de la région, le groupe des promoteurs a commencé à travailler sur la sélection de semences de maïs pour améliorer les rendements et assurer l'alimentation des familles paysannes. À cette époque, la production de maïs atteignait entre 800 et 900 kilos par hectare, assurant ainsi seulement $40 \%$ de la consommation familiale. Dans ce contexte, le groupe de promoteurs s'est penché sur les savoir-faire paysans qui aidaient à mieux mener la sélection et l'amélioration des semences, et à diffuser ces savoir-faire à travers la méthodologie « Paysan à Paysan » (Campesino a Campesino, MCaC).

Grâce à ces efforts, les rendements dans les parcelles ont augmenté. La production moyenne a atteint ainsi entre 3000 et $4000 \mathrm{~kg} / \mathrm{ha}$, et a permis d'assurer l'alimentation des foyers. Cependant, au-delà de l'augmentation des rendements, il est aussi important de noter que le travail mené dans les communautés a permis de revaloriser les variétés locales de maïs, et de préserver celles qui répondaient le mieux aux besoins locaux. Par ailleurs, l'achat de maïs n'étant plus nécessaire, les foyers ont perçu une amélioration de leur économie. Finalement, notons qu'en améliorant la qualité de 1 'alimentation, ces initiatives ont également eu un impact positif sur la santé des familles paysannes. (Figure 3)

Figure 3 : Inventaire de variétés de maïs locales de Tlaxcala

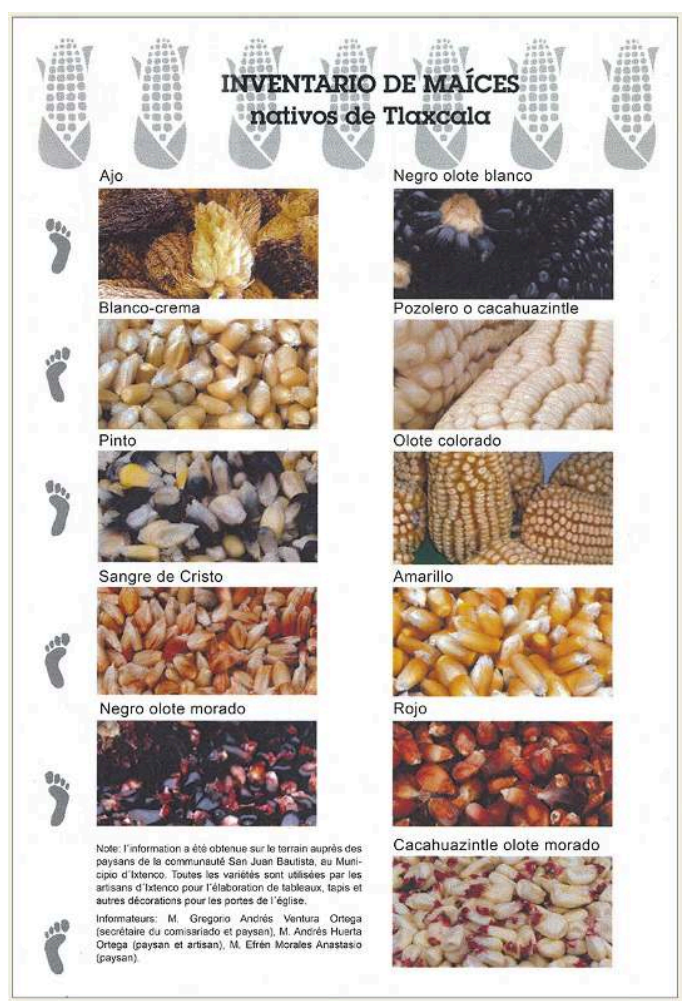

14 Cette expérience a enrichi les méthodes traditionnellement mobilisées. Aux techniques apprises au Guatemala, les promoteurs ont intégré les savoir-faire locaux de leur région et, par la suite, ont pu réaliser des stratégies globales pour la conservation des sols et des eaux, pour l'augmentation de la fertilité des parcelles par la rotation et 
l'association des cultures, pour l'utilisation d'engrais verts, de plantes de couverture et d'engrais organiques. Grâce à cela, la production de maïs a augmenté et les variétés paysannes ont été préservées; on a également promu le reboisement de la région avec des espèces locales, la culture du maguey, du nopal et d'arbres fruitiers. Outre les bénéfices économiques, tout cela a permis de récupérer la biodiversité dans les parcelles familiales.

Mais en même temps que ces initiatives étaient mises en œuvre dans les parcelles de Tlaxcala, l'équipe des promoteurs s'est donné pour but de tisser des liens avec d'autres organisations similaires. Ainsi, à la fin de 1980, le SEDEPAC a établi un projet conjoint avec l'Union Nacional de Agricultores y Ganadero (UNAG) du Nicaragua pour instaurer un programme d'échanges et de formations. Sur l'aspect méthodologique, il est intéressant de remarquer que lors de ces échanges, le concept "Paysan à Paysan " $(\mathrm{CaC})$ et ses procédés d'échange horizontal des savoir-faire entre les paysans, a été formalisé.

Quelque temps plus tard les promoteurs se sont séparés du SEDEPAC à la suite de problèmes institutionnels. Bien que cela n'ait impliqué ni la fin des échanges avec les paysans centraméricains, ni la fin des ateliers et programmes qu'ils développaient à Tlaxcala, cette situation conflictuelle a réduit la disponibilité de financement et, en conséquence, la fréquence de leurs activités. Ainsi, à partir de cet événement, le noyau des promoteurs a dû repenser ses formes de travail pour se procurer des financements et poursuivre son action. Sur cette base et avec les revenus que le groupe obtenait grâce à l'exploitation de ses propres parcelles, les promoteurs ont établi des relations de travail avec différentes organisations dans les États de Veracruz, Hidalgo, Puebla et Guanajuato, pour les conseiller pendant quatre ans.

Le groupe des promoteurs a également participé à la coordination des réunions «Paysan à Paysan» tenues au Mexique en 1990 et 1991, au Salvador en 1992, au Nicaragua en 1993, au Guatemala en 1994, au Honduras en 1995, à Cuba en 1997 et au Costa. Rica en 1998. Il a également poursuivi l'expérimentation des techniques de collecte de l'eau, la diversification des arbres fruitiers et d'autres espèces locales, l'utilisation de barrières vivantes, l'incorporation de chaume et d'engrais naturels, parmi d'autres techniques, pour enrichir le sol des parcelles. Sur le plan méthodologique, ils ont continué à consolider les méthodes $\mathrm{CaC}$ et la planification participative.

En 1993, le groupe de promoteurs de Vicente Guerrero a présenté à l'agence de coopération "Pain pour le monde» (Pan Para el Mundo) un plan de travail pour plusieurs communautés de la région nord-ouest de Tlaxcala. La proposition a été approuvée pour un an et, à partir de ce projet, le groupe a pris le nom de Grupo Vicente Guerrero (GVG) en raison de leur communauté d'origine. Après cela, une phase de restructuration du groupe a commencé, ainsi que l'association d'autres paysans et communautés aux initiatives.

19 Un nouveau projet est né. Des foires de maïs ont été organisées pour créer des espaces d'échange et de rencontre entre les communautés. Ces foires visaient aussi à faire connaître les différentes variétés de maïs criollo récoltées chaque année par les agriculteurs, à discuter des sujets liés aux problèmes agricoles régionaux et nationaux, ainsi qu'à partager les diverses expériences paysannes autour de la conservation et de 1 'amélioration des variétés criollas de maïs. 


\section{Actions pour la protection et la défense de la diversité du maïs local dans l'État de Tlaxcala}

Selon certaines définitions, défendre signifie résister par divers moyens à l'action $\mathrm{d}$ 'agents extérieurs nuisibles. C'est dans ce sens que le GVG s'est engagé pour la défense des variétés locales de maïs qui persistent à Tlaxcala face aux semences hybrides promues par la révolution verte, et face aux semences transgéniques que le pays cherche à légaliser. À cet égard, outre les risques que les semences transgéniques font courir à l'économie paysanne et à la santé des consommateurs, le GVG a dénoncé les risques que ce maïs pose pour la diversité génétique de cette espèce.

En ce sens, il faut souligner que la diversité génétique est donnée par le nombre total de caractéristiques génétiques qui se trouve dans chaque espèce, et que cette diversité est fondamentale pour assurer la capacité d'adaptation des espèces à des changements environnementaux (pluie ou sécheresse). Ainsi, les espèces avec une faible diversité génétique ont une plus grande vulnérabilité face à ces changements. Lorsque la taille des populations se réduit, la diversité génétique s'érode et les races, les variétés et les caractéristiques morphologiques importantes pour l'agriculture paysanne, tendent à se perdre (Kato Yamakake et al. 2009). Par conséquent, la conservation de la diversité est $d$ 'une très grande importance et doit être basée sur le maintien des pratiques et usages qui la permettent, ainsi que sur la préservation des races elles-mêmes.

Dans le cas du maïs et d'autres plantes cultivées, le terme de race est utilisé pour regrouper des individus ou des populations qui partagent des caractéristiques communes d'ordre morphologique, écologique, génétique et culturelle qui permettent de les différencier d'autres groupes. Les races partagent une distribution géographique et climatique définie, ainsi qu'une histoire évolutive commune. Chacune peut comprendre de nombreuses variétés qui se différencient les unes des autres par la forme de l'épi, la couleur et la texture des graines, leur processus d'adaptation et leur diversité génétique (Wellhausen et al. 1951).

Cependant, il faut également contribuer à la préservation des pratiques et savoirs sociaux qui permettent le maintien et la diversification de ces caractéristiques (couleur, texture, composition et apparence). Ainsi, les techniques d'évaluation et d'amélioration des variétés que pratiquent les communautés paysannes et indigènes, leur permettent d'obtenir des variétés adaptées aux conditions climatiques défavorables, aux parasites et ravageurs et aux besoins des communautés. Pour cette raison, il faut faire très attention à la façon dont les paysans et les communautés indigènes identifient et nomment leurs variétés de maïs : camauac maïs jaune, cacahuacintle maïs aux graines similaires aux fèves de cacao, conejo petit maïs, cuarenteño maïs blanc de 40 jours, tigre maïs qui pousse dans la brousse, cañuela maïs dur avec un cycle de trois mois. Un autre élément à observer est le respect des coutumes ancestrales et la réalisation de rituels dans les périodes de semailles et de récolte. Dans ce même sens, il faut suivre les conseils des aînés ainsi que prendre en compte les cycles lunaires et donc, semer et récolter trois jours avant ou après la pleine lune (Sandoval et al. 2003).

24 Face à cette diversité d'éléments symboliques et matériels, le GVG s'est engagé à la défense du maïs paysan, mais aussi à celle des savoir-faire et rituels associés à sa culture. Pour ce faire le Groupe organise des foires annuelles autour des diverses variétés des maïs paysans ainsi que d'autres espèces, des réunions et ateliers de 
formation, des initiatives pour tisser des liens avec les secteurs académiques, sociaux et gouvernementaux afin de promouvoir la préservation des semences indigènes et des savoir-faire paysans.

Concernant les foires, il faut mentionner que ce sont des espaces de rencontre et d'échange de semences de maïs, mais aussi d'autres plantes, haricots, fèves et courges parmi $d$ 'autres espèces importantes dans le régime alimentaire des communautés. En outre, les foires ont aussi servi d'espaces d'échanges d'expériences sur les techniques de conservation et de reproduction des semences paysannes, la création de banques communautaires de semences, ainsi que pour diffuser l'information sur les effets des semences transgéniques pour la santé, l'environnement et les variétés natives de maïs, auprès du grand public (Figures 6 et 7). Jusqu'en 2016, 19 foires ont été organisées dans la communauté Vicente Guerrero, et d'autres dans les États de Oaxaca, Guerrero, Michoacán, Chiapas, Puebla et Quintana Roo. (Figures 4 et 5)

Figure 4 : Échanges de semences

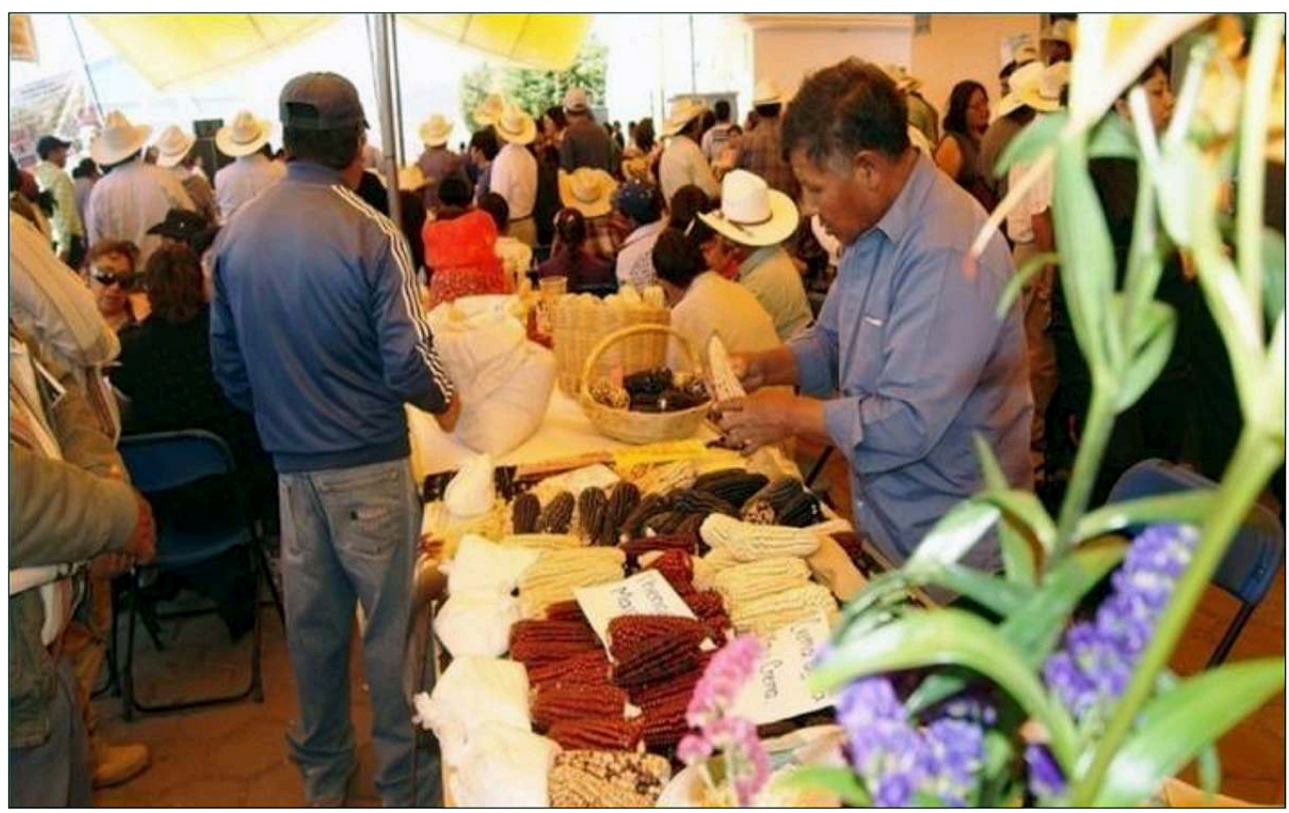


Figure 5 : Réunion sur les semences paysannes

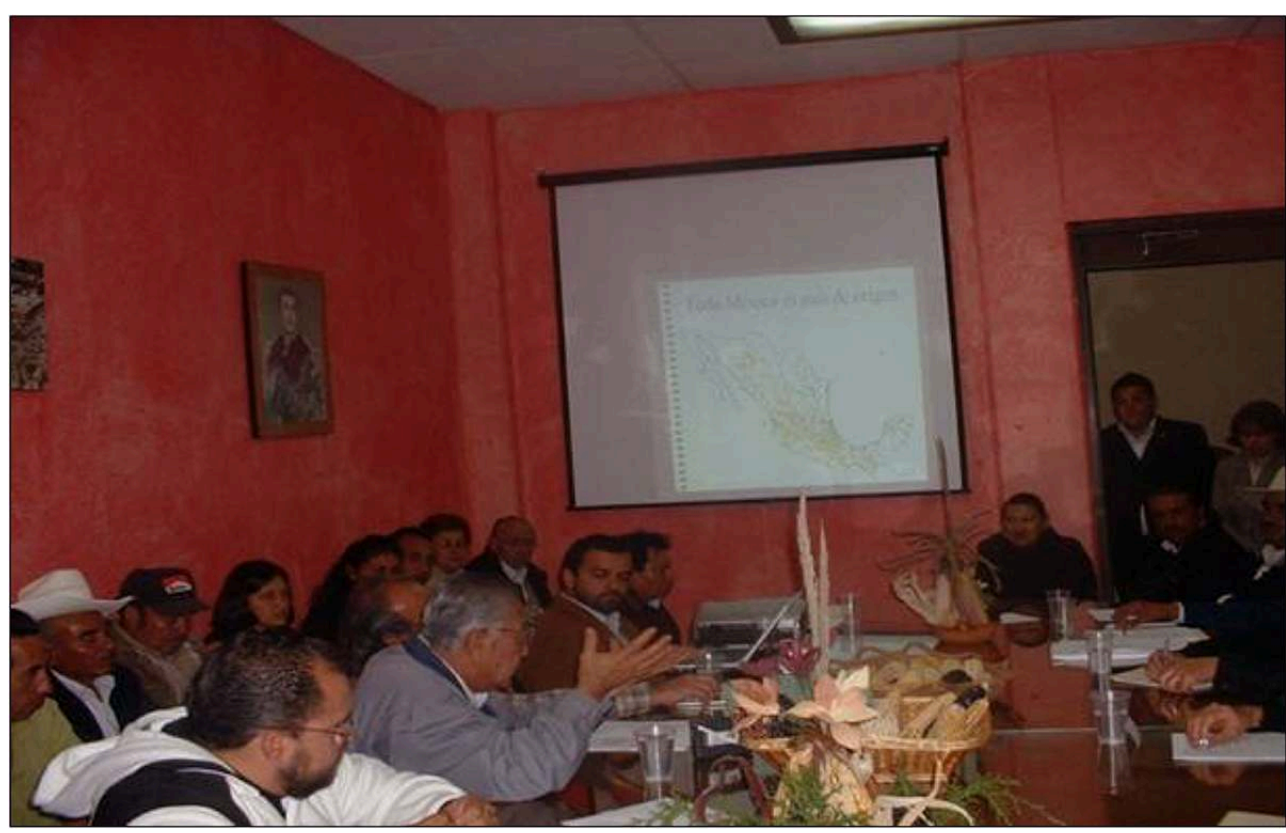

D'autre part, soulignons les efforts déployés par le GVG dans le domaine législatif pour créer des instruments juridiques qui aident à protéger le maïs indigène dans l'État. Ce fut un long cheminement qui a commencé en 2006. D'abord ont été organisées des réunions pour élaborer le projet de loi et, par la suite, il a fallu amener l'initiative au sein de l'Assemblée pour la soumettre à discussion et approbation.

La première réunion a eu lieu le 9 juin 2006 à l'Institut technique de l'agriculture et de l'élevage (Instituto Tecnológico Agropecuario). Étant donné que cette institution offre aux ingénieurs agronomes une formation en agriculture conventionnelle, il a été proposé d'y discuter sur les risques posés par l'agriculture intensive et extensive, sur les alternatives agro-écologiques et, finalement, sur l'importance du maïs indigène pour la souveraineté alimentaire du pays. Plus tard, le 23 septembre 2006, une deuxième réunion a été organisée à l'intérieur du Congrès de l'État, où le GVG a expliqué aux députés la nécessité d'un instrument juridique pour protéger le maïs natif de Tlaxcala face aux semences OGM. De multiples idées ont été proposées à cette occasion pour élaborer un projet de loi.

Le 22 mars 2007 a eu lieu la troisième réunion dans les locaux du Congrès de l'État, pour discuter les initiatives que les paysans et les autorités ejidales des Mairies de Nanacamilpa, Tepetitla, Calpulalpan, Benito Juárez et Españita avaient préparées. (Figures 6 et 7). À la rédaction finale de ce projet de loi ont aussi participé un avocat et un député d'opposition de la LIX législature. De plus, grâce à la participation de chercheurs et de plusieurs organisations sociales de Tlaxcala et de Puebla, ce projet de loi s'est beaucoup enrichi. Cependant, l'approbation de ce projet de loi a dû attendre jusqu'à la rentrée législative suivante, début 2008. (Figures 8 et 9) 
Figure 6 : Foire du maïs

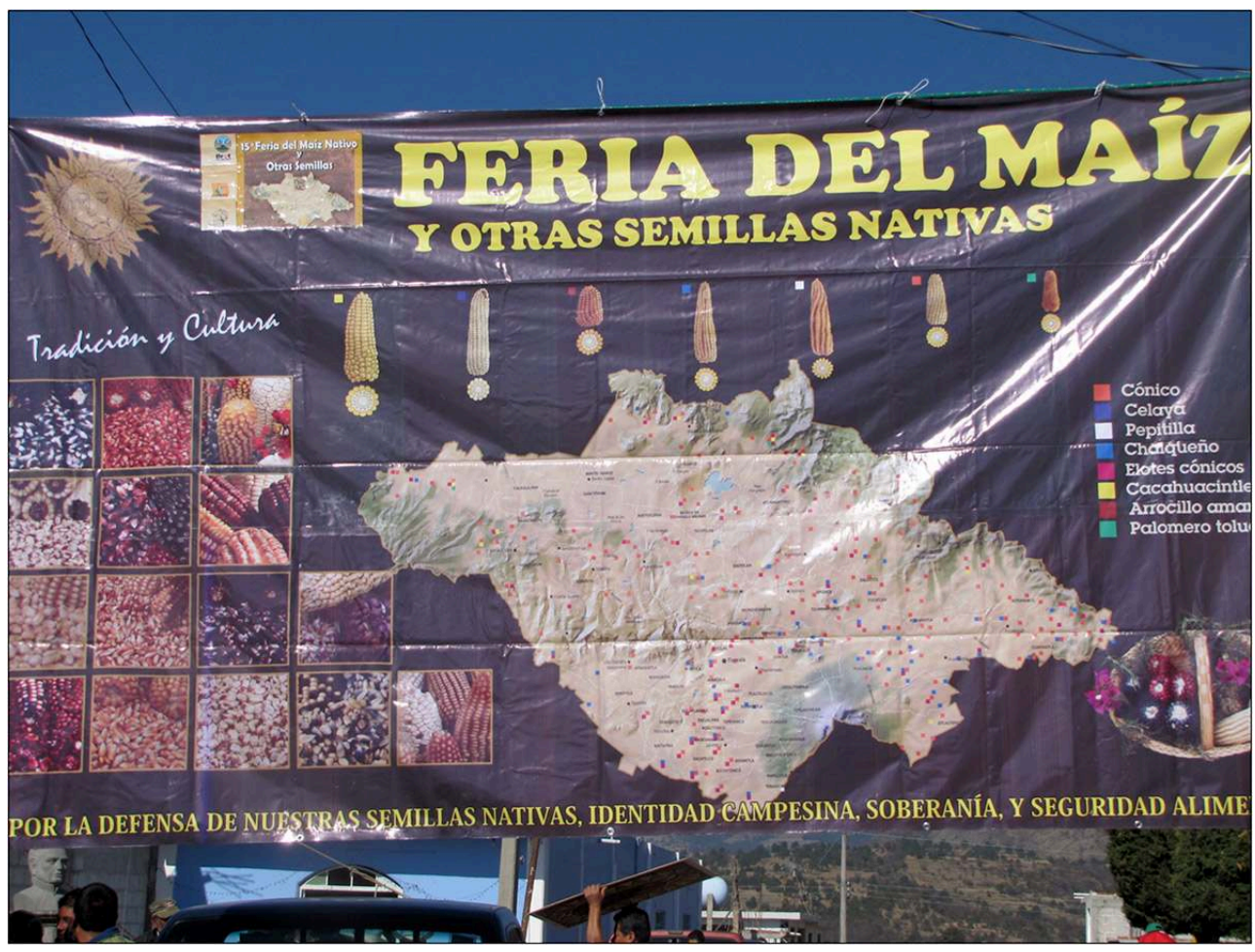

Figure 7 : Foire du maïs

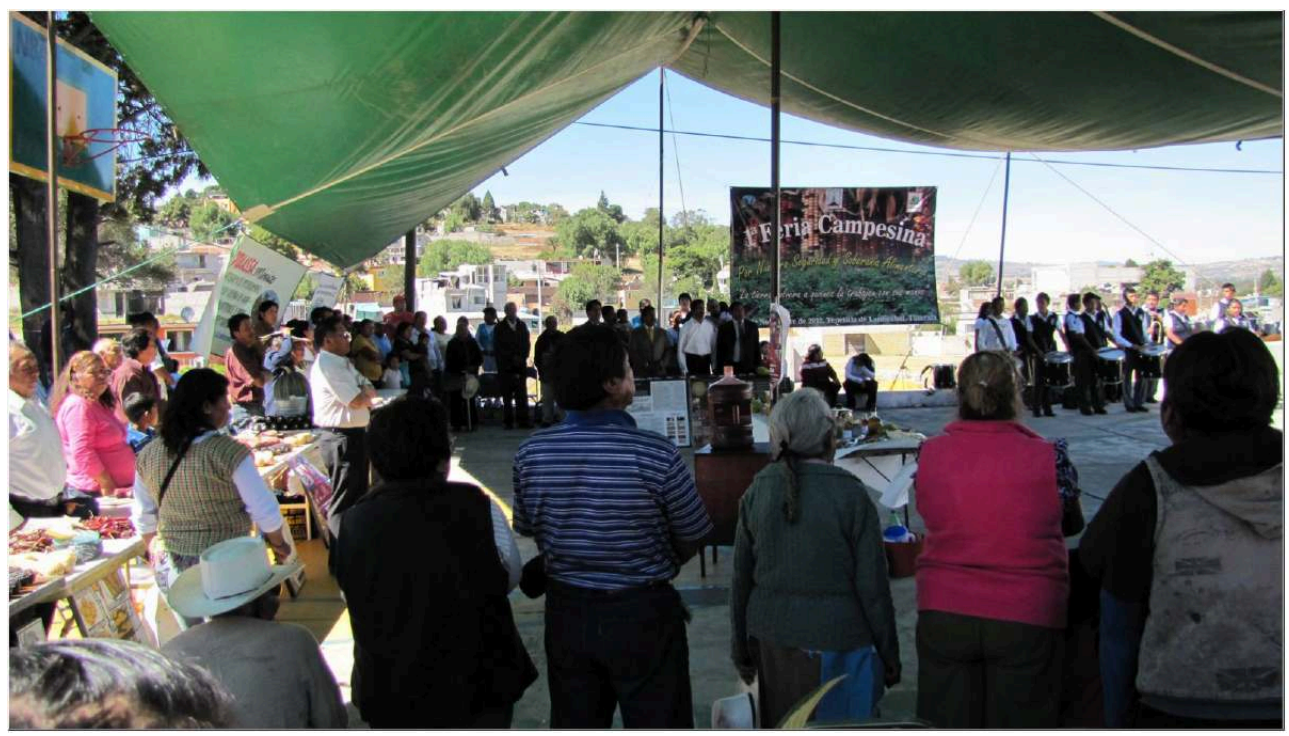


Figure 8 : Forum pour la défense du maïs

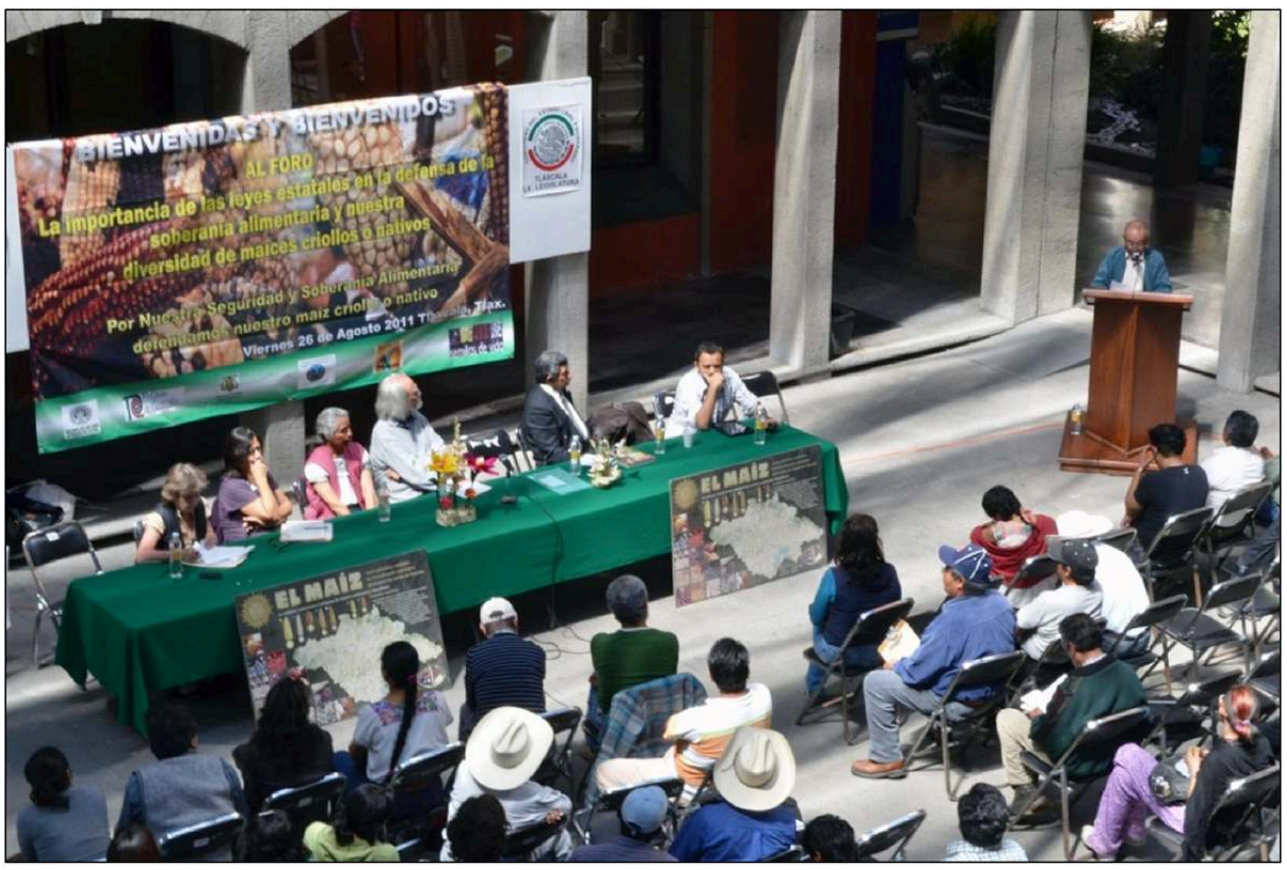

Figure 9 : Forum pour la défense du maïs

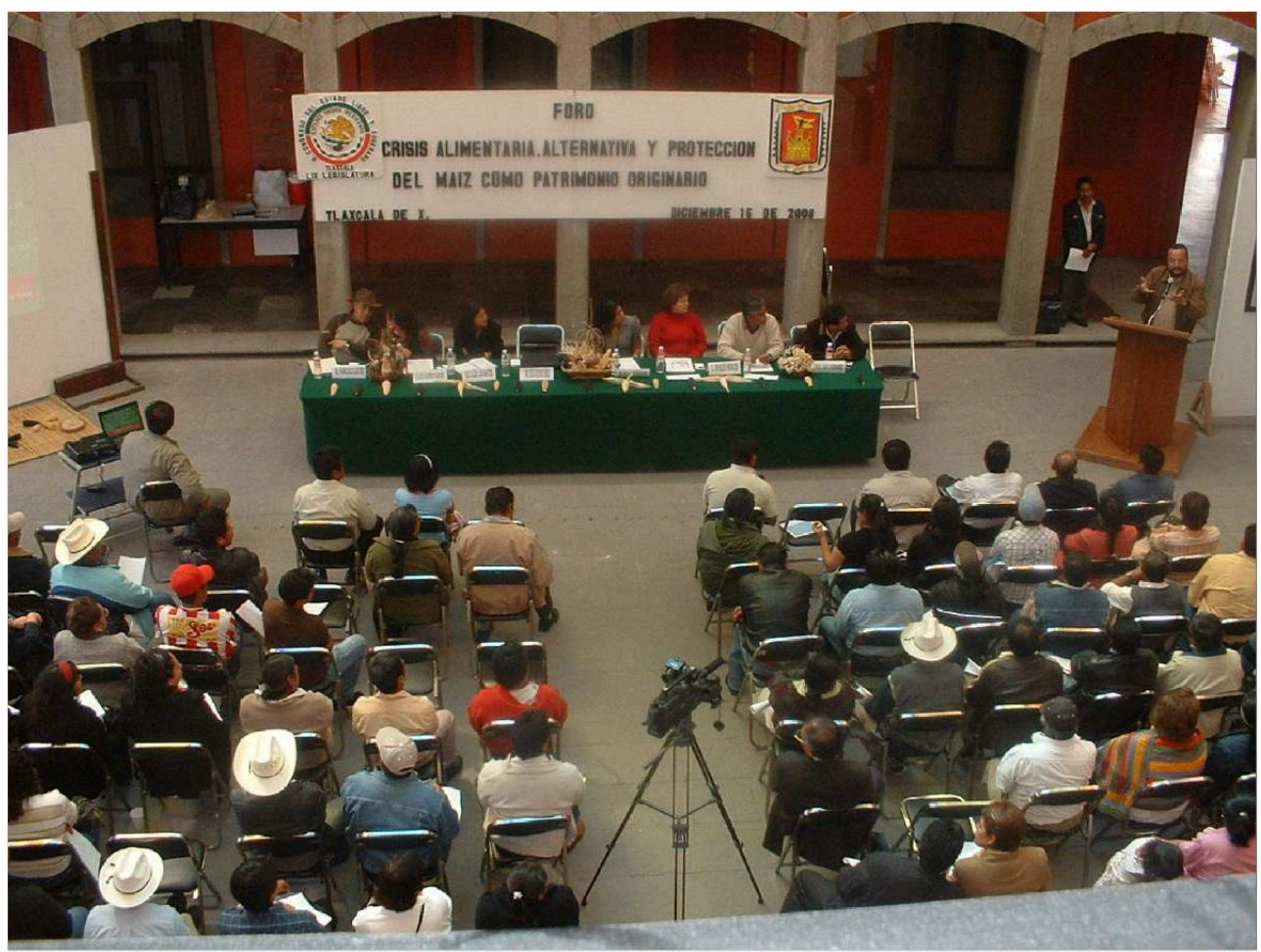

Une quatrième réunion s'est tenue le 18 octobre 2008 avec les équipes juridiques du Congrès de l'État afin d'harmoniser le texte avec les cadres légaux du niveau fédéral, tout en conservant les arguments issus des communautés qui revendiquent la valeur alimentaire, productive et culturelle du maïs natif ${ }^{1}$. (Figures 10 et 11) 
Figure 10 : Superficie ensemencée avec les cinq cultures les plus importantes dans l'État de Tlaxcala entre 1990 et 2006

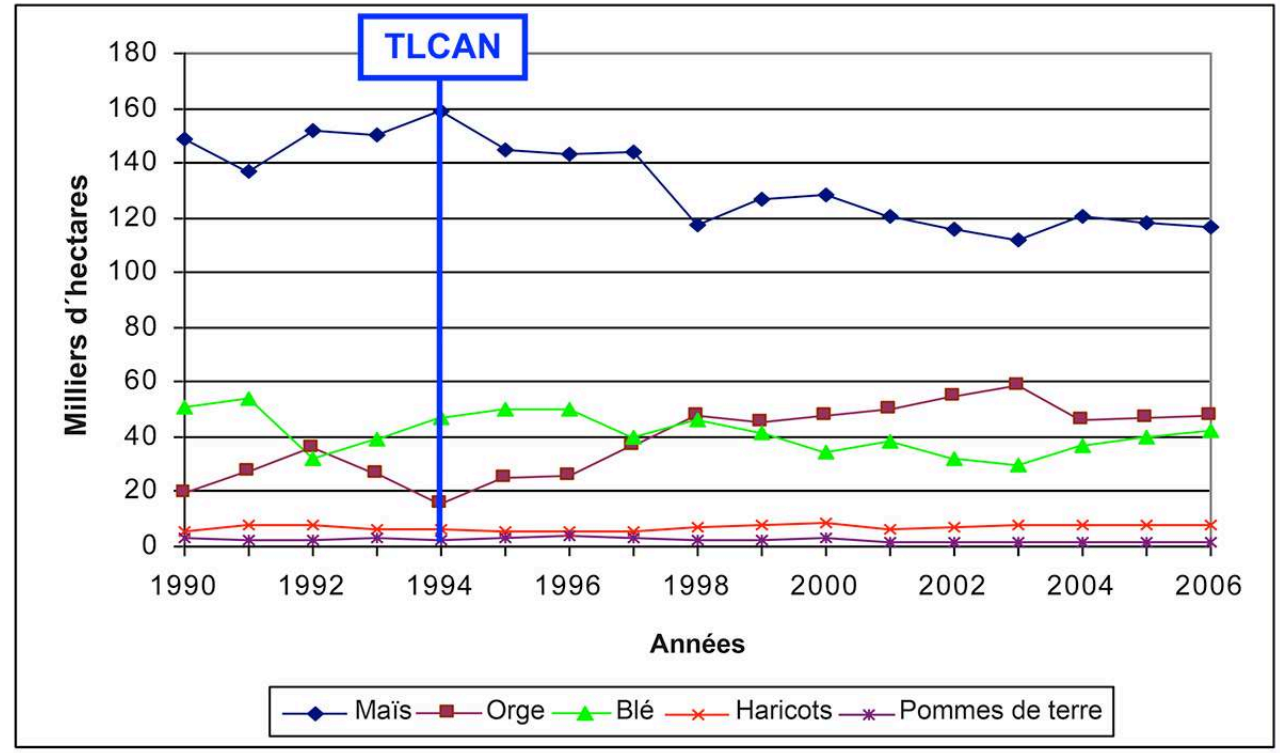

Source : Élaboration de l'auteur avec les données du Système d'Information Agro-alimentaire pour Consultation (SIACON), SIAP, 2007

Figure 11 : Superficie ensemencée entre 1990 et 2006 dans l'État de Tlaxcala

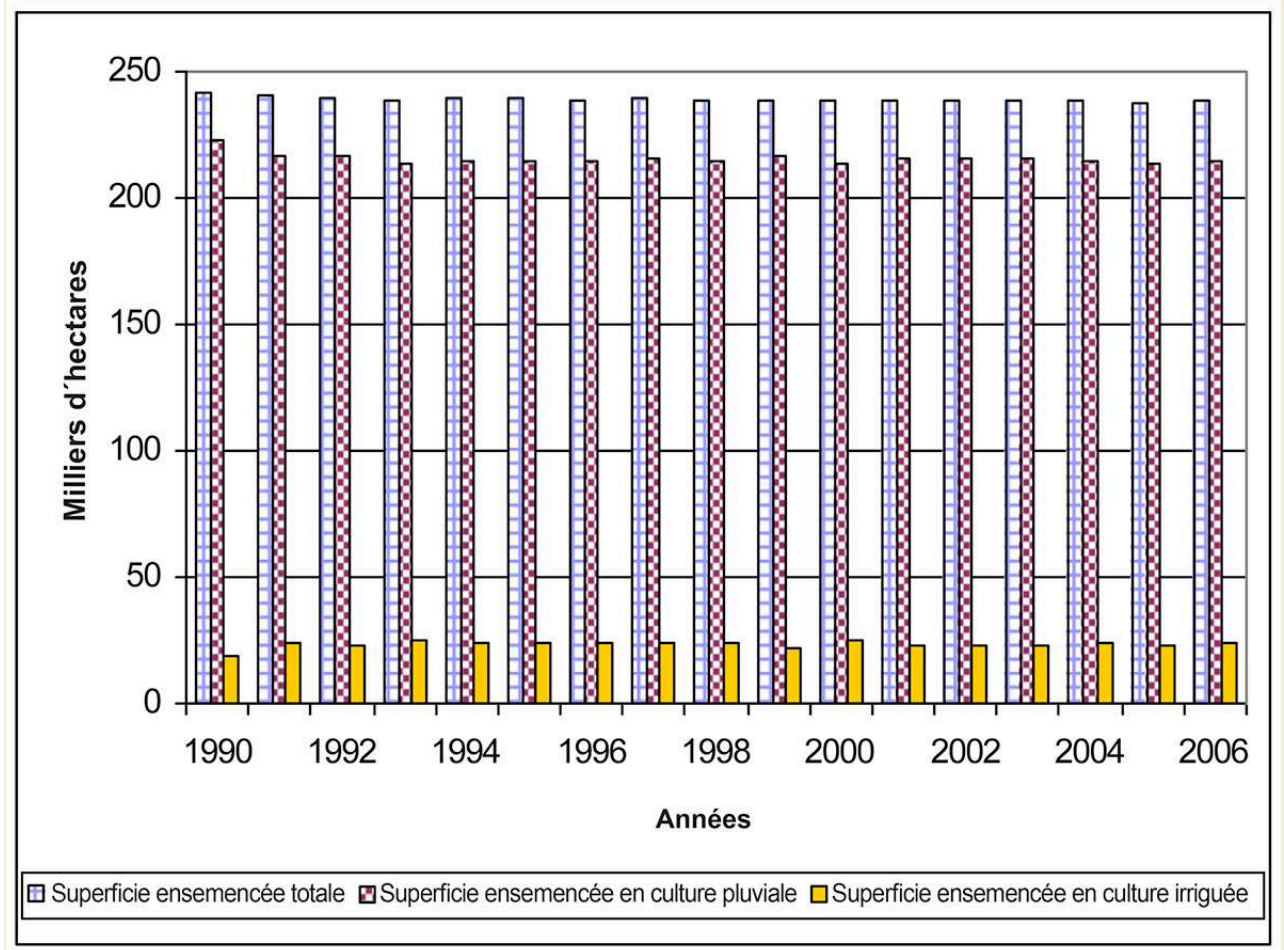

30 Cependant, dans l'immédiat, les résultats n'ont pas été à la hauteur des espérances. Le Comité de révision a entravé le processus et le GVG a dû redéfinir de nouvelles stratégies pour accélérer l'approbation du projet de loi. Un appel a été lancé pour tenir une assemblée populaire au sein du Congrès avec la participation de divers acteurs sociaux et faire pression sur les autorités. Dans ce cadre, s'est tenue l'«Assemblée 
d'État du Maïs » Asamblea Estatal del Maíz à laquelle s'est associé le «Groupe Local de l'Incidence Politique de Tlaxcala ", Grupo Local de Incidencia Política Tlaxcallan (GLIP-T) pour faire le suivi des accord pris à cette occasion.

31 Cependant, de façon parallèle aux travaux menés au Congrès, des stratégies complémentaires ont été déployées. Par exemple, diverses autorités des communautés telles que les Commissariats, Comisariados, les conseils de vigilance et les ejidatarios, ont organisé des assemblées pour décréter, conformément aux droits qui leur sont assurés par la Constitution, leurs ejidos comme des territoires libres d'OGMs. Par la suite, plusieurs organisations paysannes et sociales se sont solidarisées et ont aidé à faire pression sur les autorités chargées de promulguer la loi qui assurait la protection du maïs natif face aux semences transgéniques.

\section{Les résultats et les actions à venir}

Les actions déployées tout au long de ce processus ont aidé à mieux informer les communautés sur les risques posés par le maïs transgénique. Dans ce sens, le GVG a alerté sur le fait que la contamination des variétés locales pouvait entraîner la perte de la diversité génétique, et que cette contamination pouvait aussi être coûteuse pour les paysans en raison des droits de propriété tenus par les sociétés transnationales sur les OGM. L'accent a été mis sur la dépendance qu'elles provoquaient car les paysans doivent les acheter à chaque cycle agricole en plus des intrants chimiques, dans un marché aux prix très fluctuants.

Il est donc important de signaler qu'au lendemain des assemblées et grâce aux actions de pression menées, ainsi qu'à l'initiative de la députée Ana Lilia Rivera au sein du Congrès local, les diverses Commissions législatives responsables ont validé le projet de loi et approuvé la «Loi agricole de protection du maïs comme patrimoine originel en diversification constante et alimentaire dans l'État de Tlaxcalla » Ley Agrícola de Fomento y Protección del Maíz como Patrimonio Originario, en DIversificación Constante y Alimentario, para el Estado de Tlaxcala) le 13 janvier 20112.

34 Une fois la loi publiée dans le journal officiel, il a été décidé de renforcer le GLIP-T avec d'autres membres pour déployer des campagnes de communication sur les contenus de cette nouvelle loi. C'est ainsi que l'information a été donnée aux ejidos et aux communautés de l'État, mais aussi diffusée à travers les médias et présentée dans des réunions, foires et autres espaces publics. De plus, un appel a été lancé pour réaliser la Seconde Assemblée Départementale pour élaborer une loi règlementaire pouvant assurer l'applicabilité de la loi récemment approuvée, et définir les stratégies à suivre pour empêcher l'entrée et l'usage des semences OGM dans l'État.

Actuellement le GLIP-T est un groupe composé de paysans, scientifiques, étudiants, consommateurs et autorités communautaires et ejidales de l'État de Tlaxcala, mais aussi d'autres villes de Puebla, Hidalgo, Oaxaca, Michoacán et de la ville de Mexico. Il a pour fonction de rendre compte de la situation qu'induite le processus juridique qui, jusqu'à maintenant, bloque l'utilisation du maïs transgénique dans le pays. En outre, le groupe a la responsabilité de l'organisation de conférences et d'ateliers pour apprendre à identifier les plants de maïs transgéniques et éviter de les confondre avec les hybrides. 


\section{Conclusions}

Au-delà de l'approbation de la loi mentionnée, les initiatives déployées par le GVG ont atteint d'autres objectifs. En premier lieu, il faut souligner l'intégration des comités communautaires à diverses localités, ce qui a favorisé l'organisation et la mobilisation sociale autour des questions agricoles. De même, grâce aux foires et réunions organisées, le GVG a réussi à tisser un réseau d'échanges et de solidarités qui va au-delà de Tlaxcala. Ces espaces de rencontre favorisent la mise en commun des expériences agricoles, ainsi que l'organisation sociale pour faire face à différents enjeux.

D'un autre côté, l'exemple du GVG a encouragé d'autres organisations à initier des actions similaires pour la défense et la protection de leurs variétés natives de maïs. Pour ce faire les foires de maïs se tiennent de plus en plus souvent dans divers ejidos et municipalités du pays. Ces efforts aident à régénérer le tissu social des communautés, ainsi qu'à renforcer une dynamique d'échange de semences et de savoir-faire entre les peuples. Ainsi, il est clair qu'au-delà des initiatives juridiques, le plus important pour assurer la préservation de la biodiversité cultivée, c'est de continuer à cultiver les semences de ferme et de ne pas adopter les OGM. D'après l'expérience du GVG, il est possible de dire que les dispositions légales interdisant les OGM sont très utiles mais ne suffisent pas. La participation des paysans reste indispensable pour assurer la conservation des maïs natifs.

Enfin, il convient de noter que la création de la loi pour protéger les variétés locales de maïs à Tlaxcala a encouragé d'autres États à faire de même. Au Michoacán, par exemple, l'initiative a été lancée par un parti politique d'opposition au sein du Congrès. Dans l'État de Morelos, l'initiative est venue par le travail de groupes citoyens en relation avec des chercheurs et des paysans. Bien que, dans chaque cas, les circonstances et les itinéraires soient différents, il est important de prêter attention à ces différentes expériences pour arriver à articuler une stratégie à l'échelle nationale, car bien qu'un tribunal fédéral ait émis une interdiction de la culture du maïs transgénique, celle-ci reste provisoire et, en conséquence, le risque continue d'exister.

En tout état de cause, l'expérience montre que, outre les instruments juridiques, la meilleure manière de s'opposer aux semences transgéniques et de limiter l'érosion des ressources génétiques locales, est de revendiquer et de promouvoir l'agriculture paysanne et ses savoir-faire sur les usages de la biodiversité. En ce sens, il faut demander aux institutions gouvernementales des programmes pour encourager l'usage des semences paysannes, ainsi que des pratiques plus saines pour la production de nos aliments, en particulier du maïs et des haricots.

«Il est temps de défendre ce qui nous appartient, notre agriculture, notre patrimoine, notre souveraineté alimentaire, notre culture, notre maïs »

\section{BIBLIOGRAPHIE}

Congreso del Estado de Tlaxcala, expediente parlamentario 142/2008. 
Documentos del Grupo Vicente Guerrero. En ligne : http:www.vicenteguerrero.org.mx

Kato Yamakake T.A., Mapes Sánchez C., Mera Ovando L.M., Serratos Hernandez J.A. \& Bye Bottler R.A. 2009 - Origen y diversificación del maíz. Una revisión analítica. , México D.F., Universidad Nacional Autónoma de México, Comisión Nacional para el Conocimiento y Uso de la Biodiversidad.

Kato Yamakake T.A., Ortega Paczka R., Boege E., Wegier A., Serratos Hernández J.A., Alavez V., Jardón-Barbolla L., Moyers L. \& Vecchyo D. 2013 - Origen y diversidad del maíz. In : ÁlvarezBuylla E. \& Piñeyro Nelson A. (Ed.), El Maíz en peligro ante los transgénicos. México D.F., UNAM, UCCS, Universidad Veracruzana : 25-60.

Massieu Trigo Y. 2016 - El maíz en Tlaxcala: cultura, alimentación y políticas públicas La Jornada del campo 105, 18 de junio de 2016.

Muñoz Orozco A. 2005 - Centli-maíz, prehistoria e historia del maíz. Chapingo, Colegio de Posgraduados.

Ortega Paczka R. 2003 - La diversidad del maíz en México. In : Esteva G. \& Marielle C. (Ed.), Sin maíz no hay país. México D.F., CONACULTA : 123-154.

Ortiz Báez P. A., Muñoz Lara L.O. y Briones J.E. 2016 - Maíces para la vida. Cultura y biodiversidad en Tlaxcala. La Jornada de Oriente, 4 de marzo de 2016. http://www.lajornadadeoriente.com.mx/ 2016/03/04/maices-para-la-vida-cultura-y-biodiversidad-en-tlaxcala/

Sandoval M., Esteva G. \& Marielle C. 2003 - El maíz y los pueblos indios. In : Sin maíz no hay país. México, CONACULTA : 59-66.

Wellhausen E.J., Mangelsdorf P.C., Roberts L.M. \& Hernández Xolocotzi E. 1951 - Razas de maíz en México: su origen, características y distribución. México, oficina de Estudios Especiales, Secretaría de Agricultura y Ganadería de México.

\section{NOTES}

1. Documents du Grupo Vicente Guerrero : http:www.vicenteguerrero.org.mx

2. Assemblée de l'État de Tlaxcala, Dossier parlementaire 142/2008; Documents du Grupo Vicente Guerrero : http:www.vicenteguerrero.org.mx

\section{RÉSUMÉS}

L'article présente les stratégies suivies par le Groupe Vicente Guerrero à Tlaxcala pour empêcher l'entrée et diffusion du maïs transgénique dans la région, ainsi que pour revitaliser l'utilisation des variétés locales. L'histoire du groupe Vicente Guerrero et de ses principaux promoteurs est suivie dès 1990 jusqu'à l'approbation en 2011 de la « Loi de promotion et de protection du maïs comme patrimoine originaire, en constante diversification et alimentaire pour l'État de Tlaxcala". Sur la base de ces éléments, l'article analyse l'importance de la défense juridique de la diversité cultivée, de même que les actions sociales qui contribuent à la conservation in situ des variétés locales. 
The article presents the strategies deployed by the Vicente Guerrero Group in Tlaxcala to prevent the use of transgenic corn in the region, as well as to revitalize the use of native varieties of corn. It also shows the history of the group in question and the itineraries followed by the nucleus of peasant promoters from the 1990s until the approval of the "Law for the promotion and protection of corn as original heritage, in constant diversification and food for the state of Tlaxcala" in 2011. Based on these elements, we discuss the importance of both the legal defense of cultivated diversity, and the social actions that help in the in situ conservation of native varieties.

El artículo expone las estrategias desplegadas por el Grupo Vicente Guerrero en Tlaxcala para impedir el uso de maíces transgénicos en la región, así como para revitalizar el uso de las variedades nativas de maíz. Se presenta la historia del grupo en cuestión y se da cuenta de los itinerarios seguidos por el núcleo de promotores campesinos desde los años 1990 hasta la aprobación de la "Ley de fomento y protección al maiz como patrimonio originario, en diversificación constante y alimentario para el estado de Tlaxcala" en 2011. A partir de esos elementos se reflexiona sobre la importancia tanto de la defensa jurídica de la diversidad cultivada, como de las acciones sociales que ayudan a la conservación in situ de las variedades criollas.

\section{INDEX}

Index géographique : Mexique

Palabras claves : maíces criollos, organización campesina, Tlaxcala, Metodología Campesino a Campesino

Keywords : native maize, peasant organization, Tlaxcala, Metodología Campesino a Campesino

Mots-clés : maïs criollos, organisation paysanne, Tlaxcala, Metodología Campesino a Campesino

\section{AUTEUR}

\section{PÁNFILO HERNÁNDEZ ORTIZ}

Membre de l'association civile Proyecto de Desarrollo Rural Integral - Calle Domingo Arenas S/N Vicente Guerrero, Municipio Españita, Tlaxcala. Mail: guerrerogv@prodigy.net.mx 